\title{
The Distal Oblique Bundle of the Distal Interosseous Membrane of the Forearm
}

\author{
Hisao Moritomo, MD, $\mathrm{PhD}^{1}$ \\ ${ }^{1}$ Department of Physical Therapy, Osaka Yukioka College of Health \\ Science, Yukioka Hospital Hand Center, Osaka, Japan
}

J Wrist Surg 2013;2:93-94.

The distal interosseous membrane (DIOM) of the forearm is on the distal side of the central band of the interosseous membrane, spanning the radius and ulna at the dorsal region of the pronator quadratus muscle. Recent anatomical studies have revealed that the thickness of the DIOM varies widely among specimens $(0.5-2.6 \mathrm{~mm})$, and an obvious thick fiber within the DIOM, called the distal oblique bundle (DOB), exists in $40 \%$ of specimens. ${ }^{1,2}$ The DOB originates from the distal ulna $54 \mathrm{~mm}$ (on average; range, $50-57 \mathrm{~mm}$ ) proximal to the ulnar head and runs distally to insert on the dorsal

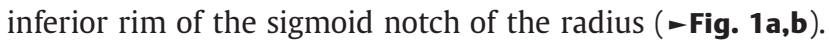
The DOB has an anatomical relationship with the triangular fibrocartilage complex (TFCC) (-Fig. 2), both of which probably work as isometric collateral ligaments of the distal radioulnar joint (DRUJ) because the ulnar insertions of the
Address for correspondence Hisao Moritomo, MD, PhD, Department of Physical Therapy, Osaka Yukioka College of Health Science, Yukioka Hospital Hand Center, 2-2-3, Ukita, Kita-ku, Osaka-shi, Osaka 5300021, Japan (e-mail: moritomo@tcct.zaq.ne.jp).

DOB and the deep limbs of the radioulnar ligament almost coincide with the course of the axis of forearm rotation. ${ }^{3}$

The TFCC is the primary soft-tissue DRUJ stabilizer, and the DIOM acts as a secondary stabilizer of the DRUJ when the dorsal and palmar radioulnar ligaments of the TFCC are cut., ${ }^{4,5}$ In normal situations, the influence of the DIOM on DRUJ stability is relatively inconsequential. However, after a TFCC injury, an ulnar head resection, or a Sauvé-Kapandji procedure, the DIOM has a more important role in the stability of the ulnar head or ulnar stump. The clinical relevance of the DIOM includes its stabilizing effect on the DRUJ in ulnar shortening procedures. Recent study has revealed that ulnar shortening with the osteotomy performed proximal to the attachment of the DIOM has a more favorable effect on stability of the DRUJ as compared to distal osteotomy,
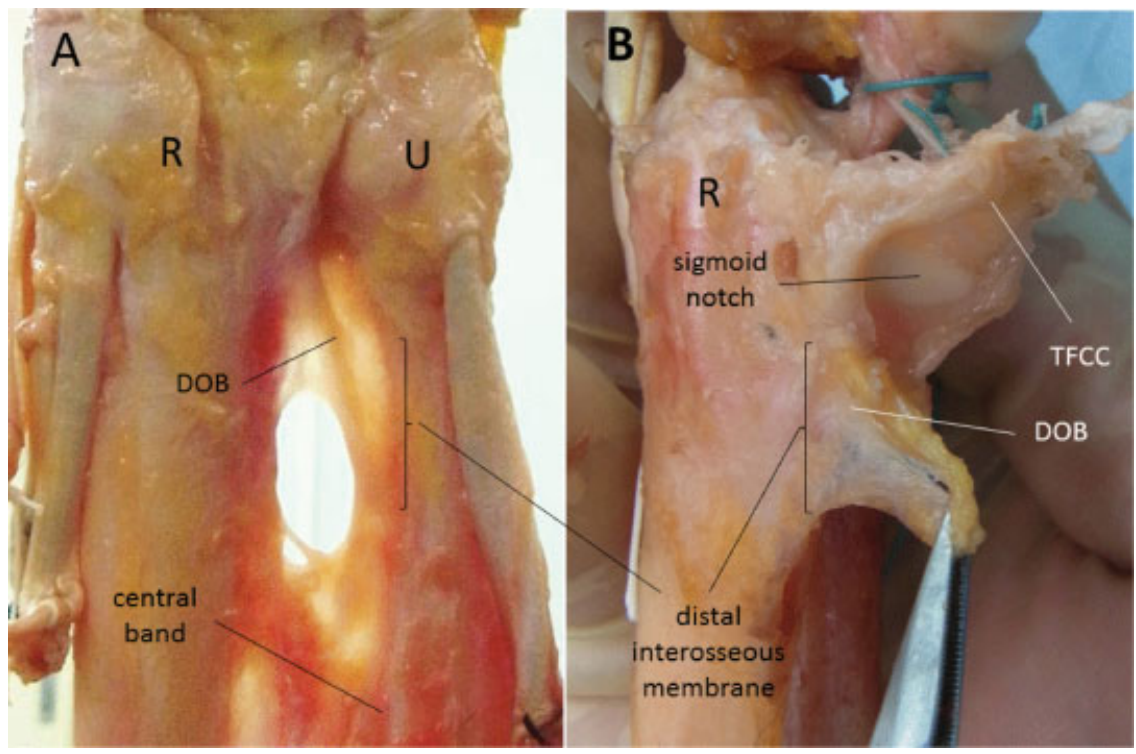

Fig. 1a,b Distal interosseous membrane of the right cadaver wrist. (a) Dorsal view of the distal oblique bundle (DOB) and the central band. (b) The distal radius with the distal interosseous membrane and TFCC viewed from the dorsoulnar side. The ulna has been removed. DOB inserts on the dorsal inferior rim of the sigmoid notch of the radius. $R$, radius; $U$, ulna. 

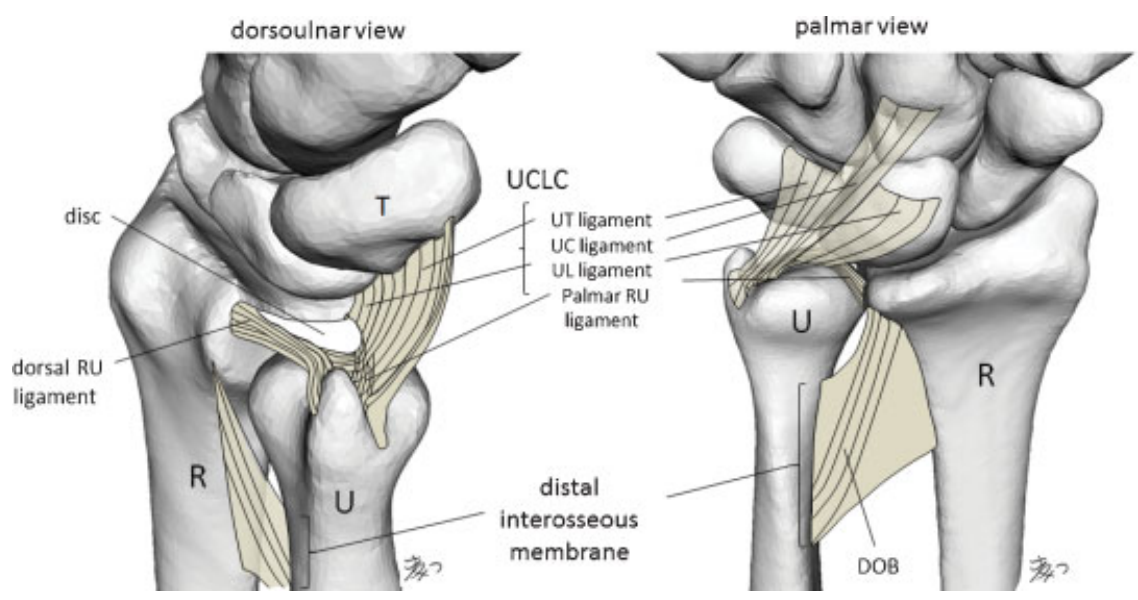

Fig. 2 Anatomical relationship between DOB and TFCC, which comprises the ulnocarpal ligamentous complex (UCLC), disk, and palmar and dorsal radioulnar (RU) ligaments. UT, ulnotriquetral ligament; UC, ulnocapitate ligament; UL, ulnolunate ligament.

especially in the presence of a DOB. ${ }^{6}$ The longitudinal resistance to ulnar shortening is significantly greater in proximal shortening than in distal shortening. These results suggest that proximal ulnar shortening in the presence of a robust DIOM could be a risk factor for nonunion, and for patients with ulnocarpal abutment but no instability of the DRUJ, it may be advantageous to perform a distal osteotomy.

\section{References}

1 Noda K, Goto A, Murase T, Sugamoto K, Yoshikawa H, Moritomo H. Interosseous membrane of the forearm: an anatomical study of ligament attachment locations. J Hand Surg Am 2009;34(3): 415-422

2 Kitamura T, Moritomo H, Arimitsu S, et al. The biomechanical effect of the distal interosseous membrane on distal radioulnar joint stability: a preliminary anatomic study. J Hand Surg Am 2011;36(10):1626-1630

3 Moritomo H, Noda K, Goto A, Murase T, Yoshikawa H, Sugamoto K. Interosseous membrane of the forearm: length change of ligaments during forearm rotation. J Hand Surg Am 2009;34(4): 685-691

4 Watanabe H, Berger RA, Berglund LJ, Zobitz ME, An KN. Contribution of the interosseous membrane to distal radioulnar joint constraint. J Hand Surg Am 2005;30(6):1164-1171

5 Kihara H, Short WH, Werner FW, Fortino MD, Palmer AK. The stabilizing mechanism of the distal radioulnar joint during pronation and supination. J Hand Surg Am 1995;20(6): 930-936

6 Arimitsu S, Moritomo H, Kitamura T, et al. The stabilizing effect of the distal interosseous membrane on the distal radioulnar joint in an ulnar shortening procedure: a biomechanical study. J Bone Joint Surg Am 2011;93(21):2022-2030 